\title{
The First Infrared Parallaxes for T-Dwarfs
}

\author{
C. G. Tinney \\ Anglo-Australian Observatory, PO Box 296. Epping. 1710 Australia.
}

A. Burgasser

UC Los Angeles, 8371 Mathematical Sciences, CA.

J. D. Kirkpatrick

Infrared Processing \& Analysis Centre, Caltech, Pasadena, CA

\begin{abstract}
We present first results from the first parallax program to use an infrared camera to target the observation of a sample of methane T-dwarfs, using the ESO $3.5 \mathrm{~m} \mathrm{NTT}$.
\end{abstract}

\section{The Methane Brown Dwarfs}

In recent years several groups have announced the discovery of numerous field counterparts to the extremely cool methane brown dwarf Gl 229B (Burgasser et al. 1999, 2002ab; Strauss et al. 1999; Leggett et al. 2000; Tsvetanov et al. 2000; Cuby et al. 2000). The extremely low photospheric temperatures of these "T-dwarfs" $(900-1000 \mathrm{~K})$ mean that their atmospheres are dominated by the effects of dust and methane formation - neither of which are amenable to simple modelling. The discovery of sizable numbers of T-dwarfs, means that we are now in a position to empirically determine the the locus of T-dwarf cooling curves, without being forced to rely on poorly constrained model atmospheres. The discovery of several objects by SDSS with spectra intermediate between the L- and T- spectral types (e.g. Leggett et al. 2000) is particularly exciting, as parallaxes for these objects will show exactly where, on the brown dwarf cooling curve, the L-T transition occurs.

Trigonometric parallaxes are also essential to understanding the space density of T-dwarfs. Luminosity function estimates for T-dwarfs (e.g., . Burgasser et al. 1999) are currently based on the assumption that they are single. Recent programs targeting more luminous L-dwarfs (Martín et al. 1999; Koerner et al. 1999), have shown that $30 \%$ of objects observed sufficiently intently, are found to be binaries. If this trend continues into the T-dwarf regime, a luminosity function based on colour-magnitude relations would be meaningless. Trigonometric parallaxes are essential to determine the actual luminosities of these objects and whether they are single or binary, so that a meaningful luminosity function for T-dwarfs can be constructed. 

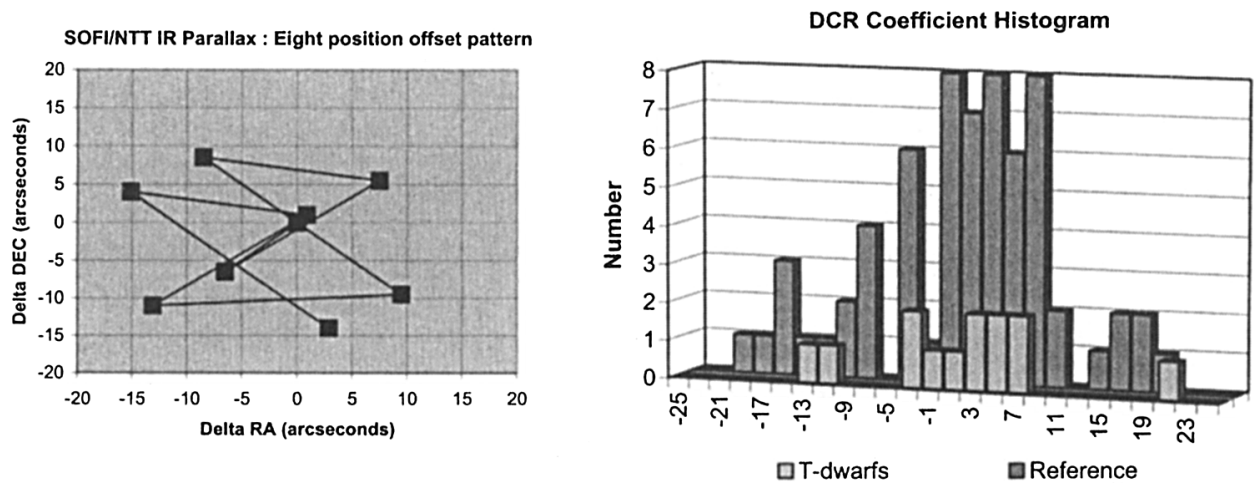

Figure 1. Left panel - Fixed dither pattern used for all epoch observations. Offsets are in arcseconds from the base pointing position. Right panel - DCR coefficient histograms for reference stars and programme T-dwarfs, in units of mas/Tan(Zenith Angle) (Tinney 1993,1996).

\section{Parallaxes and the Infrared}

Traditional parallax techniques based on photography are completely unable to target objects as faint and red as T-dwarfs. CCD parallax work in the optical at the USNO, ESO and Palomar (Tinney 1996; Tinney et al. 1995; Monet et al. 1992; Dahn et al. 2002) have indicated that parallaxes can be obtained for objects as faint as $\mathrm{I}=18-19$ at distances $\$ 70 \mathrm{pc}$. However, this still leaves the T-dwarf class of objects (with I $>21$ ) unobservable. To date only a few of the very brightest and closest T-dwarfs have proved measurable (Dahn et al. 2002).

Over the last two years, therefore, we have been extending our experience in optical astrometry into the infrared, where the $\mathrm{J}<16$ magnitudes of most of the detected T-dwarfs make progress possible. Indeed, there are a several reasons to prefer the infrared for high precision astrometry.

First, the effects of differential colour refraction (the different amount of refraction the atmosphere produces in red target stars, compared to blue reference stars) are much reduced by working at longer wavelengths. Second, seeing improves in the infrared, leading to smaller images, and smaller amounts of differential seeing. Thirdly, as we show below, the stringent requirements on maintaining control of observations at constant hour angles (at least for T-dwarfs) is not present in the infrared. This considerably increases the flexibility and efficiency of infrared parallax observing, over the optical. And of course, finally, T-dwarfs are much brighter, compared to the sky in the near-infrared than in the optical. In fact, this ratio is highest in the J-band, and so it was here that all our astrometric effort has been focussed.

Infrared parallax observations were pioneered by Jones (2000), who targetted the extremely active (and unfortunately also quite distant at $76 \mathrm{pc}$ ) very late M-dwarf PC0025+0447 and the nearby M-dwarf VB10. Our NTT program represents the first publication of results for a major program targetting a significant sample of brown dwarfs in the infrared. 

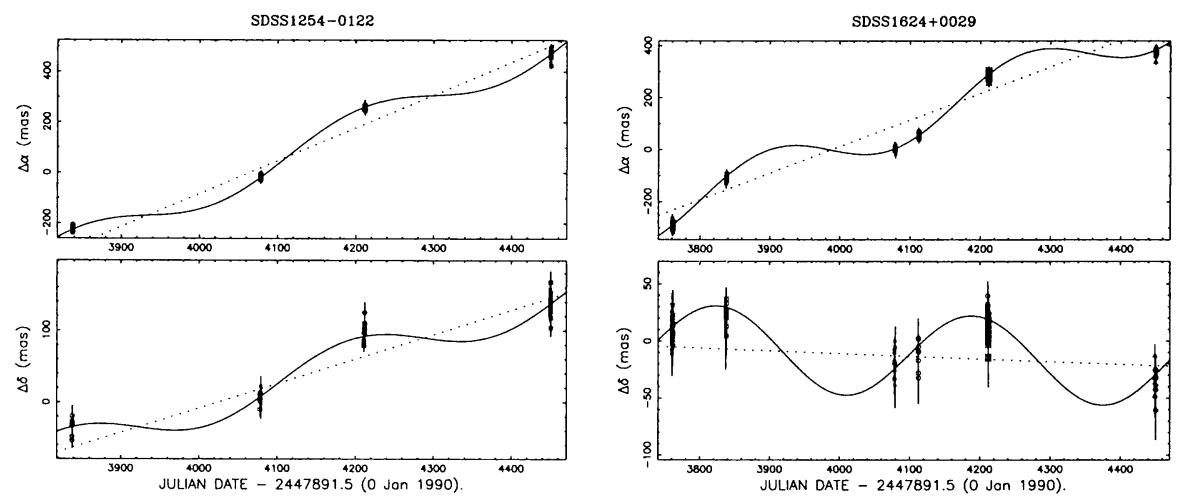

Figure 2. The panels show plots of the components of motion across the sky as a function of time. The dotted line is the proper motion of the T-dwarf, and the solid line is the parallax plus proper motion.

\section{The Programme}

Observations have been carried out at 3 epochs per year over the last two years with the SOFI infrared camera on the ESO $3.5 \mathrm{~m}$ New Technology Telescope (NTT). SOFI was used in its "large field" mode in which it provides a 5 ' $\times 5$ field-of-view with 0.292 " pixels.

Exposures of each target were acquired with a fixed dither pattern (Fig. 1 (left)) as eight 120 s exposures. This exposure pattern meant that together this 16 minutes of exposure time sampled many different interpixel spacings. As much as was feasible, given observing time constraints, we attempted to acquire all epoch observations at the same hour angle as the very first epoch observation acquired, so as to minimise DCR effects. As well as these epoch observations, all targets were also observed as they rose and set, so that DCR calibrations for each target could be developed.

An interesting result of these calibrations was to find that T-dwarfs have effective wavelengths in the J-band which are essentially indistinguishable from the ensemble of background reference stars against which their positions are measured. This is shown in Fig. 1 (right), which plots histograms of the DCR coefficients determined for both reference stars and programme T-dwarfs. The similarities in the ensemble values are clear. (These coefficients were derived using the method described in Tinney $1993 \& 1996$. Typical uncertainties in the individual determinations are $\approx \pm 2-6$.) As a result, for T-dwarfs at least, infrared observations are not rigidly tied to being carried out near the meridian, which will add greatly to the flexibility and efficiency of IR parallax programmes.

\section{The Programme and Some Results}

Our parallax programme targetted ten T-dwarfs (2MASS 0559-14, 1047+21, $1217-03,1225-27,1346-00,1534-29,1546-33$ and SDSS 1021-03, 1254-01, $1624+00)$, for which we present results for two here. Figure 2 shows plots of 
the parallax and proper motion fits we have obtained for SDSS1254-01 and SDSS1624+00. For SDSS 1254-01 we find $\pi=73.2 \pm 1.9$ mas, $\mu=491.0 \pm 2.5$ $\mathrm{mas} / \mathrm{y}, \theta=285 \pm 1^{\circ}$, and for SDSS1624+00 we find $90.9 \pm 1.2 \mathrm{mas}, 373.0 \pm 1.6$ $\operatorname{mas} / \mathrm{y}, 269 \pm 1^{\circ}$ (respectively).

The USNO has published parallax estimates for three T-dwarfs: 2MASS 0559-14, SDSS1254-01 and SDSS1624+00 (Dahn et al. 2002). While all three were included in our program, insufficient epochs for 2MASS0559-14 were obtained to measure a parallax. The equivalent parallax solution quantities for those we obtained above, are given by Dahn et al. as for SDSS1254-01: $84.1 \pm$ $1.9 \mathrm{mas}, 496.1 \pm 1.8 \mathrm{mas} / \mathrm{y}, 285.2 \pm 0.4^{\circ}$, and for SDSS1624+00: $90.7 \pm 2.3 \mathrm{mas}$, $383.2 \pm 1.9 \mathrm{mas} / \mathrm{y}, 269.6 \pm 0.5^{\circ}$. These independent observations and solutions agree within uncertainties for almost all parameters - the exception being the parallax for SDSS1254-01, for which the two solutions are different by about $5-\sigma$, though Dahn et al. do comment that with only 1.2 years of data on this target their solution can only be considered preliminary.

\section{Conclusion}

We have shown that high precision parallaxes can be obtained with common-user near-infrared cameras using techniques very similar to those which have been demonstrated to work in the optical. The new generation of large format infrared imagers based on HAWAII1 $(1 \mathrm{~K})$ and HAWAII2 (2K) HgCdTe arrays, and the new generation of $1 \mathrm{~K}$ InSb arrays, offer exciting prospects for the astrometry of cool brown dwarfs in the future.

\section{References}

Cuby, J.G. et al. 2000, A\&A, 349, L41

Dahn, C.C. et al. 2002, AJ, 124, 1170

Burgasser, A. J., et al. 1999, ApJ, 522, L65

Burgasser, A. J., et al. 2000a, ApJ, 531, L57

Burgasser, A. J., et al. 2000b, AJ, 120, 1100

Jones, H.R.A. 2000, HIPPARCOS and the Luminosity Calibration of the Nearer Stars, 24th IAU General Assembly, Joint Discussion 13, Manchester, UK.

Koerner, D.W., Kirkpatrick, J. D., McElwain, M.W. \& Bonaventura, N.R. 1999, ApJ, 526, L25

Leggett, S. K., et al. 2000, ApJ, 536, L35

Martín, E.L., Brandner, W. \& Basri, G. 1999, Science, 283, 1718

Monet et al. 1992, AJ, 103, 638

Strauss, M. A., et al. 1999, ApJ, 522, L61

Tinney, C.G. 1993, AJ, 105, 1169

Tinney, C.G., Reid, I.N., Gizis, J. \& Mould, J.R., 1995, AJ, 110, 3014

Tinney, C.G. 1996, MNRAS, 281, 644

Tsvetanov, Z. I., et al. 2000, ApJ, 531, L61 